FIAT MONEY IN A PAIRWISE-TRADING, MULTI-GOOD, OVERLAPPING GENERATIONS MODEL

\author{
by \\ Yasuo Maeda \\ Discussion Paper No. 250, October 1989
}

\author{
Center for Economic Research \\ Department of Economics \\ University of Minnesota \\ Minneapolis, Minn 55455
}




\title{
Fiat Money in a Pairwise-Trading, Multi-Good, Overlapping Generations Model
}

\author{
Yasuo Maeda* \\ Department of Economics \\ University of Minnesota \\ Minneapolis, Minnesota 55455
}

September, 1989

Abstract: This paper studies a stationary, pure exchange overlapping generations model with a particular kind of randomly generated pairwise trading pattern. This model, in contrast to the usual overlapping generations models, has the following implications. As regards existence, it is shown that a standard boundary condition on preferences is sufficient to imply the existence of a stationary monetary equilibrium. As regards properties of the equilibrium, for some specifications, my model has a stationary equilibrium in which claims bear a higher rate of return than fiat money. It is also proved that this stationary equilibrium is conditionally Pareto optimal.

* I am grateful for helpful comments and discussions on this paper by Neil Wallace, Timothy Kehoe, Karl Shell, Yves Balasko, David Levine, Hidehiko Ichimura, Shinsuke Nakamura and participants at seminars at Cornell University, the University of Pennsylvania, the University of Western Ontario, the University of California at Riverside and the University of Minnesota. 


\section{Introduction}

This paper studies a stationary, pure exchange overlapping generations model with a particular kind of randomly generated pairwise trading pattern: essentially, one type of old agent meets one type of young agent at each date where types vary in terms of endowments and preferences over three distinct goods.

This model is to be compared with the usual overlapping generations model - the same model with centralized meetings. As is well-known, in the centralized version, fiat money can have value only if its rate of return is as high as that of any other asset. One consequence is that stringent conditions on preferences, endowments, and intertemporal technologies are required in order for there to be an equilibrium with valued fiat money. And, of course, the centralized version cannot produce an equilibrium in which fiat money has a lower rate of return than other assets. In contrast, the model with pairwise meetings requires only a standard boundary condition on preferences in order for there to be an equilibrium with valued fiat money. Also, in some versions, it is consistent with some assets bearing a higher rate of return than fiat money. In the centralized version, stationary monetary equilibria are Pareto optimal. An analogous result is shown to hold for the pairwise meeting version. Thus, the rate of return discrepancies in the pairwise trading model are not symptoms of non-optimality - as they are in other models of fiat money.

There are other models which use pairwise trading (for example, Feldman (1973), Ostroy (1973), Ostroy and Starr (1974), Harris (1979) and Kiyotaki and Wright (1988)). All these models except for the model of Kiyotaki and Wright are models for commodity money or inside money and focus on the optimality of pairwise trading process. The model of Kiyotaki and Wright can make fiat money circulate and the setting of their model is similar to my model. In their model, fiat money circulates because they assume that there is no cost to store fiat money and there is cost to store commodities. They investigate the model with a game theoretic approach and analyze a Nash equilibrium. In this paper, I use a choice theoretic approach and seek a general equilibrium. Townsend (1987) constructed a multi-good model in which fiat money and interest-yielding capital coexist at equilibrium. Ile introduced pairwise meetings in the model with long-lived agents.

In Section 2, the model is constructed. First the characteristics of agents are described. Then the pairwise trading pattern is described. Section 3 defines a monetary equilibrium and establishes the existence of stationary monetary equilibrium. Section 4 discusses rate of return discrepancies between money and another asset. Section 5 establishes the conditional Pareto optimality of stationary monetary equilibrium.

\section{The model}

\section{The characteristics of agents}

Consider an overlapping generations model in which every young agent lives two periods. There are three types of young agents in the model; type 1 agents, type 2 agents and type 3 agents. Agents of the same type are homogeneous with respect to preferences, endowments and discount factors. There are three consumption goods; good 1, good 2 and good 3. 
In each period, one young agent of each type is born. Hence three young agents are born altogether. The agent of type $i$ derives utility from good $i$ and good $i+1$ when he is young and old. (The addition in terms of $i$ is interpreted as modulo 3; i.e., when $i=3, i+1=1$.) Denote his one-period utility function as $U^{i}\left(c^{i}, c^{i+1}\right)\left(U^{i}: \Re_{+}^{2} \longrightarrow \Re\right)$; where $c^{i}$ is the amount of good $i$ and $c^{i+1}$ is the amount of good $i+1$. The agent of type $i$ is endowed with good $i$ when he is young and old. Denote his endowment by $\left(w^{i}, \dot{w}^{i}\right) \in \Re_{+}^{2}$; where $w^{i}$ is the endowment of good $i$ when he is young and $\dot{w}^{i}$ is the endowment of good $i$ when he is old. To simplify the model, we assume symmetry among agents in the sense that $U^{i} \equiv U$ for all $i$ and $\left(w^{i}, \dot{w}^{i}\right) \equiv(w, \dot{w})$ for all $i$. In period $t=1$, there is one initial old agent of each type. Hence there are three initial old agents altogether. The initial old agent has the one-period utility function corresponding to his type. The initial old agent of type $i$ is endowed with $\dot{w}$ units of good $i$ and $\bar{M}$ units of fiat money.

\section{Pairwise trading}

We assume there are three isolated markets; market 1 , market 2 and market 3 . Trading takes place as follows. In period $t=1$, the initial old agent of type $i$ is located at the market $i$. In each period, each young agent visits one of the markets depending on the result of the random device. The random device generates the number 0 with probability $\pi$ and the number 1 with probability $1-\pi$. We consider the following simple random meeting; if the result of the random device is 0 then the young agent of type $i$ visits the old agent of type $i$, if the result of the random device is 1 then the young agent of type $i+1$ visits the old agent of type $i$. It is assumed that this probability is known to all agents and that they maximize their expected utilities using this probability.

\section{Roles of fiat money}

Suppose that the old agent of type $i$ meets the young agent of type $i+1$ in period $t$. In this case the old agent wants good $i+1$ which the young agent is endowed with. However, the young agent does not want good $i$ which the old agent is endowed with. This is what Jevons (1875) called a single coincidence of wants. If fiat money is acceptable to the young agent and if the old agent has fiat money then the old agent can get good $i+1$ in exchange for fiat money. Anticipating this possibility, the young agent will accept fiat money and that is what gives rise to a monetary equilibrium.

If we assume that all agents who are alive get together to trade, the centralized market structure, then exchange can occur as follows without the use of money. First, the old agent of type $i$ who wants good $i+1$ can get good $i+2$ from the young agent of type $i+2$ who wants good $i$. Then, he can get good $i+1$ from the young agent of type $i+1$ in exchange for the good $i+2$. Therefore, there may not exist a monetary equilibrium in the centralized version.

\section{Claims}

We consider claims as assets other than fiat money. Claims are pieces of paper saying that the issuer of this claim promises to give certain units of certain good to the person who holds this claim in the next period. There are three goods in the economy. Therefore, three types of claims can be considered; a promise to give good $i$, a promise to give good $i+1$, and a promise to give good $i+2$. However, in the market where a young agent of type $i$ visits, only person who can supply claims is the young agent of type $i$. Since the young agent of type $i$ is only endowed with good $i$, only claim we can consider meaningfully is the claim which promise to give certain units of good $i$. In the model, the young agent of type $i$ is the only one who supplies and at the same time only one who demands this claim, at equilibrium the amount of net trade of this claim is zero. However, we can still determine the rate of return of this claim. 


\section{A monetary equilibrium}

In general, prices of goods at a particular market in a particular period depend on the whole history of the economy; who meets who at each market in each period. However the simple stationary structure of my model allows me to seek a stationary equilibrium such that prices of goods at each market depend only on the type of the young agent who visits that market. Moreover, because of symmetry among agents, we can focus on a particular stationary equilibrium in the sense that, for all $i \in\{1,2,3\}$, at any market where the young agent of type $i$ visits the price of good $i$ is equal to $p^{*}$, the price of good $i-1$ is equal to $p^{* *}$ and the rate of return of the claim which promises to give good $i$ is $r^{*}$.

At the market where the young agent of type $i$ visits, the type of the old agent is $i$ or $i-1$. Since neither agent has good $i+1$ in this case, we just put the price of this good to infinity to make sure no one would demand this good, or we can assume that there is no market for this good. (Hence in the following optimization problem, we can put $y=0$ and $\dot{y}(0)=0$ in the budget constraint.)

Each agent solves the same form of the problem:

$$
\begin{aligned}
& \text { Maximize } U(x, y)+\pi \beta U(\dot{x}(0), \dot{y}(0))+(1-\pi) \beta U(\dot{x}(1), \dot{y}(1)) \\
& \text { subject to } p^{*} x+M+p^{*} b=p^{*} w \\
& y=0 \\
& p^{*} \dot{x}(0)=p^{*} \dot{w}+M+p^{*} r^{*} b \\
& \dot{y}(0)=0 \\
& p^{* *} \dot{x}(1)+p^{*} \dot{y}(1)=p^{* *} \dot{w}+M+p^{* *} r^{*} b \\
& \left(x, y,(\dot{x}(s), \dot{y}(s))_{s \in S}, M\right) \in \Re_{+}^{7}
\end{aligned}
$$

where, for agents of type $i, x$ (resp. $y$ ) is the amount of consumption of good $i$ (resp. good $i+1$ ) when young and $\dot{x}(s)$ (resp. $\dot{y}(s)$ ) is the amount of consumption of good $i$ (resp. good $i+1)$ when old contingent on state $s$.

DEFINITION 3.1: A stationary monetary equilibrium is a set of choice variables of agents $\{x, y, \dot{x}(0)$, $\dot{y}(0), \dot{x}(1), \dot{y}(1), M, b\}$, a set of prices $\left\{p^{*}, p^{* *}\right\}$ and the rate of return of the claim $r^{*}$ which satisfy the following conditions.

(i): Given a set of prices and the rate of return of the claim, a set of choice variables of agents solves (1),

(ii): $b=0$,

(iii): $M=\bar{M}$.

The following assumptions are made.

\section{ASSUMPTION 3.1:}

(a1) $U: \Re_{+}^{2} \longrightarrow \Re$ is continuous on $\Re_{+}^{2}$, continuously partially differentiable with respect to the first argument on $\Re_{++} \times \Re_{+}$and continuously partially differentiable with respect to the second argument on $\Re_{+} \times \Re_{++}$.

(a2) $U$ is strictly monotone and strictly concave. 
(a3) $\lim _{x \rightarrow 0} D_{1} U(x, y)=\infty$ for any $y \in \Re_{+}$.

(a4) $\lim _{y \rightarrow 0} D_{2} U(x, y)=\infty$ for any $x \in \Re_{+}$.

(a5) $w>0, \dot{w} \geq 0$.

(a6) $0 \leq \pi<1$.

THEOREM 3.1: There exists a stationary monetary equilibrium under the assumptions $(a 1)-(a 6)$.

PROOF: Let $m \equiv M / p^{*}, p \equiv p^{* *} / p^{*}$ and $r \equiv r^{*}$. By substitution, we obtain

$$
\begin{gathered}
\text { Maximize } U(w-m-b, 0)+\pi \beta U(w+m+r b, 0)+(1-\pi) \beta U(\dot{x}(1), \dot{y}(1)) \\
\text { subject to } p \dot{x}(1)+\dot{y}(1)=p \dot{w}+m+p r b, \\
(\dot{x}(1), \dot{y}(1), m) \in \Re_{+}^{3} .
\end{gathered}
$$

Since (a3) and (a4) assure interior solutions for $\dot{x}(1), \dot{y}(1)$ and $m$, we can obtain the following first order conditions:

$$
\begin{aligned}
m: & -D_{1} U(w-m-b, 0)+\pi \beta D_{1} U(w+m+r b, 0)+\lambda=0 \\
\dot{x}(1): & (1-\pi) \beta D_{1} U(\dot{x}(1), \dot{y}(1))-\lambda p=0 \\
\dot{y}(1): & (1-\pi) \beta D_{2} U(\dot{x}(1), \dot{y}(1))-\lambda=0, \\
b^{i}: & -D_{1} U(w-m-b, 0)+\pi \beta r D_{1} U(w+m+r b, 0)+p r \lambda=0 \\
\lambda: & p \dot{x}(1)+\dot{y}(1)=p \dot{w}+m+p r b
\end{aligned}
$$

where $\lambda$ is the Lagrange multiplier associating with the constraint. The feasibility conditions are $m=\bar{M} / p^{*}$, $b=0$ and $\dot{y}(1)=\bar{M} / p^{*}$. Let $\bar{m} \equiv \bar{M} / p^{*}$. Then from (7), we obtain $\dot{x}(1)=\dot{w}$. Substituting these values into (3)-(6) gives the following equations. From (3) and (5),

$$
D_{1} U(w-\bar{m}, 0)=\pi \beta D_{1} U(w+\bar{m}, 0)+(1-\pi) \beta D_{2} U(\dot{w}, \bar{m}) .
$$

From (4) and (5),

$$
p=\frac{D_{1} U(\dot{w}, \bar{m})}{D_{2} U(\dot{w}, \bar{m})}
$$

From (5) and (6),

$$
r=\frac{D_{1} U(w-\bar{m}, 0)}{\pi \beta D_{1} U(w+\bar{m}, 0)+p(1-\pi) \beta D_{2} U(\dot{w}, \bar{m})} .
$$

Substituting (9) into (10) gives

$$
r=\frac{D_{1} U(w-\bar{m}, 0)}{\pi \beta D_{1} U(w+\bar{m}, 0)+(1-\pi) \beta D_{1} U(\dot{w}, \bar{m})} .
$$

Note that (8), (9), and (11) have a simple recursive structure. First we seek the value of $\bar{m}$ which satisfies (8). Then substituting this value into (9) and (10) yields the equilibrium value of $r$ and $p$. Define a function $F:(0, w) \longrightarrow \Re$ as

$$
F(m) \equiv D_{1} U(w-m, 0)-\pi \beta D_{1} U(\dot{w}+m, 0)-(1-\pi) \beta D_{2} U(\dot{w}, m)
$$


By (a3) we obtain

$$
\lim _{m \rightarrow w} F(m)=\infty
$$

By (a4) and (a6) we obtain

$$
\lim _{m \rightarrow 0} F(m)=-\infty
$$

Therefore there exists $m^{*} \in(0, w)$ such that $F\left(m^{*}\right)=0$ since $F$ is continuous on $(0, w)$. This $m^{*}$ satisfies $(8)$. Let $p^{*} \equiv \bar{M} / m^{*}$. Substituting $m^{*}$ into (9) gives the equilibrium value of $p$. Once we know the equilibrium value of $p$, we can get the value of $p^{* *}$ by using $p^{*}=\bar{M} / m^{*}$. Substituting $m^{*}$ into (11) gives the equilibrium return rate $r^{*}$. Let $c^{*} \equiv w-m^{*}$ and $\dot{c}^{*} \equiv \dot{w}+m^{*}$. Then the set of choice variables of agents $\left\{c^{*}, 0, \dot{c}^{*}\right.$, $\left.0, \dot{w}, m^{*}, M, b\right\}$, the set of prices $\left\{p^{*}, p^{* *}\right\}$ and the rate of return of the claim $r^{*}$ satisfy the condition of equilibrium.

Q.E.D.

\section{Rates of return}

In the above proof of the theorem, we obtained the own rate of return of claim as

$$
r=\frac{D_{1} U(w-\bar{m}, 0)}{\pi \beta D_{1} U(w+\bar{m}, 0)+(1-\pi) \beta D_{1} U(\dot{w}, \bar{m})} .
$$

This $r$ depends on the intertemporal structure of endowments and utility functions and can be greater than unity. Hence we obtain the following corollary.

COROLLARY 4.1: The own rate of return of claim can be greater than that of fiat money.

We investigate the situation in which the own rate of return of claims is greater than that of fiat money in more detail here. Let us assume $r>1$. From one unit of the claim which is bought by one unit of good $i$, the agent of type $i$ receives in the next period $r$ units of good $i$. On the other hand, the own rate of return of fiat money is unity. If an agent carries over one unit of fiat money then in the next period he still has one unit of fiat money.

Perhaps the clearest way to explain the possible discrepancy between the own rate of return on fiat money and the own rate of return on claims is to consider the special case $\pi=0$ which means the old agent of type $i$ always meets the young agent of type $i+1$. Imagine the situation that an outside observer is collecting data for the economy - data on prices and quantities traded in the economy. At the market in which the old agent of type $i$ and the young agent of type $i+1$ trade, the exchange between good $i+1$ which the young agent has and fiat money which the old agent has actually takes place and the price of the good is $p^{*}$. At the market in which the old agent of type $i+1$ and the young agent of type $i+2$ trade, the exchange between good $i+2$ which the young agent has and fiat money which the old agent has actually takes place and the price of the good is also $p^{*}$. At the market in which the old agent of type $i+2$ and the young agent of type $i$ trade, the exchange between good $i$ which the young agent has and fiat money which the old agent has actually takes place and the price is $p^{*}$ again. The observer repeatedly observes the same situation and may conclude the price of each good is $p^{*}$ in every period. The observer also observes the own rate of return of fiat money is one and the own rate of return of the claim is $r$ which is greater than one. He 
may conclude this situation is paradoxical. But we can understand this situation. At the market in which the old agent of type $i$ and the young agent of type $i+1$ trade the price of good $i+1$ which is actually being trading is $p^{*}$ and the price of good $i$ is hidden or cannot be observed by a "careless" observer because there is no actual trade. If we take this hidden price take into account the situation can be rationalized.

To obtain the discrepancy between the own rate of return on fiat money and the own rate of return on claims, the relative price between $p^{* *}$ and $p^{*}$ plays a role. Substituting $\pi=0$ into (10) gives

$$
r=\frac{D_{1} U(w-\bar{m}, 0)}{p \beta D_{2} U(\dot{w}, \bar{m})}
$$

where $p$ is the relative price between $p^{* *}$ and $p^{*}$, i.e., $p=p^{* *} / p^{*}$. This is the own rate of return on claims in the special case $\pi=0$. Since there is no transaction cost in this model, if the rate of return on claims is expressed in money term that would be one, i.e., there is no discrepancy between the rate of return on fiat money and the rate of return on claims. This can be shown as follows. The own rate of return on claims is $r$ means that if an agent of type $i$ buys one unit of the claim by paying one unit of good $i$ he can get $r$ units of good $i$ in the next period. When he is young the price of good $i$ in terms of money is $p^{*}$ and when he is old the price of good $i$ in terms of money is $p^{* *}$. Therefore in money term he pays $p^{*}$ units when young and he gets $r p^{* *}$ units when old. Hence in money term the rate of return on claims is $r p^{* *} / p^{*}$ or $r p$. From (12),

$$
\begin{aligned}
r p & =\frac{D_{1} U(w-\dot{m}, 0)}{p \beta D_{2} U(\dot{w}, \bar{m})} p \\
& =\frac{D_{1} U(w-\bar{m}, 0)}{\beta D_{2} U(\dot{w}, \bar{m})} \\
& =1 .
\end{aligned}
$$

The last equality comes form (8) after substituting $\pi=0$.

\section{Conditional Pareto optimality of the equilibrium}

Here I define conditional Pareto optimality of an allocation plan and prove that the stationary monetary equilibrium we obtained in Theorem 3.1 is conditionally Pareto optimal. ${ }^{(1)}$ Actually, it is proved that the stationary monetary equilibrium is conditionally Pareto optimal among all feasible allocation plans including non-stationary ones. Before defining conditional Pareto optimality, I define allocation plans.

In the economy, there exists one random device which produces 0 with probability $\pi$ and produces 1 with probability $1-\pi$ each period. If 0 is produced we call state 0 , and if 1 is produced we call state 1 . Let $S$ be the set of all states, i.e., $S \equiv\{0,1\}$. There are three types of agents, namely type 1 , type 2 and type 3 . Let $K$ be the set of all types of agents, i.e., $K \equiv\{1,2,3\}$. There are three kinds of goods, namely type 1 , type 2 and type 3 . Therefore $K$ also can be the set of all kinds of goods. Let $\mathcal{N}$ be the set of periods, i.e., $\mathcal{N} \equiv\{1,2, \cdots\}$.

DEFINITION 5.1: For each $t \in \mathcal{N}$, the set of all histories up to period $t, H_{t}$, is defined by

$$
H_{t} \equiv\left\{\left(s_{1}, s_{2}, \cdots, s_{t}\right) \mid s_{j} \in S \text { for all } j \in\{1,2, \cdots, t\}\right\}
$$

(1) Peled $(1980,1982)$ and Sakai (1988) used the same optimality concept adopted in this paper. 
DEFINITION 5.2: For each $t \in \mathcal{N}$, an allocation plan for young agents in period $t, \xi_{t}$, is defined by a function

$$
\xi_{t}: H_{t} \times K \longrightarrow \Re_{+}^{3}
$$

For each $t \in \mathcal{N}$, an allocation plan for old agents in period $t, \dot{\xi}_{t}$, is defined by a function

$$
\dot{\xi}_{t}: H_{t} \times K \longrightarrow \Re_{+}^{3}
$$

An allocation plan $\xi$ is defined by

$$
\xi \equiv\left(\xi_{t}, \dot{\xi}_{t}\right)_{i \in \mathcal{N}}
$$

An allocation plan specifies the amount of each kind of good allocated to each agent for each history. If we extend $\xi_{t}\left(h_{t}, i\right)$ as $\left(c_{t}^{1}\left(h_{t}, i\right), c_{t}^{2}\left(h_{t}, i\right), c_{t}^{3}\left(h_{t}, i\right)\right)$, then $c_{t}^{j}\left(h_{t}, i\right)$ is the amount of good $j$ allocated to the young agent of type $i$ in period $t$. If we extend $\dot{\xi}_{t}\left(h_{t}, i\right)$ as $\left(\dot{c}_{t}^{1}\left(h_{t}, i\right), \dot{c}_{t}^{2}\left(h_{t}, i\right), \dot{c}_{t}^{3}\left(h_{t}, i\right)\right)$, then $\dot{c}_{t}^{j}\left(h_{t}, i\right)$ is the amount of good $j$ allocated to the old agent who meets the young agent of type $i$ in period $t$.

To specify a particular allocation plan, we should specify the amount of each good allocated to each agent at each market. In the above definition, each market is specified by specifying the type of the young agent at that market.

DEFINITION 5.3: An endowment function for young agents $\omega: K \longrightarrow \Re_{+}^{3}$ and an endowment function for old agents $\dot{\omega}: K \longrightarrow \Re_{+}^{3}$ are defined by, for all $i \in K$,

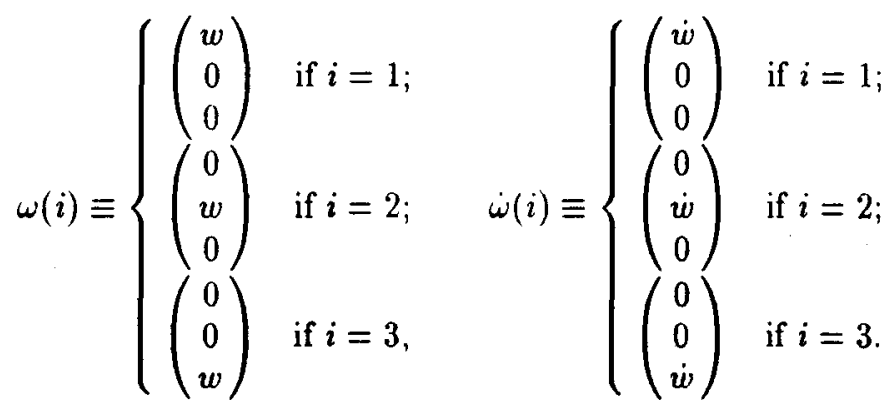

DEFINITION 5.4: An allocation plan $\xi$ is said to be feasible if for all $t \in \mathcal{N}$, for all $h_{t}=\left(s_{1}, s_{2}, \cdots, s_{t}\right) \in$ $H_{t}$ and for all $i \in K$,

$$
\xi_{t}\left(h_{t}, i+s_{t}\right)+\dot{\xi}_{t}\left(h_{t}, i\right) \leq \omega\left(i+s_{t}\right)+\dot{\omega}(i)
$$

DEFINITION 5.5: For each $i \in K$, the extended expected utility function for young agents of type $i$ is a function $\tilde{U}^{i}: \Re_{+}^{9} \longrightarrow \Re$ defined by,

for all $\left(\mathbf{c},(\dot{\mathbf{c}}(s))_{s \in S}\right) \in \Re_{+}^{9}$,

$$
\tilde{U}^{i}\left(\mathbf{c},(\dot{\mathrm{c}}(s))_{s \in S}\right) \equiv U\left(c^{i}, c^{i+1}\right)+\pi \beta U\left(\dot{c}^{i}(0), c^{i+1}(0)\right)+(1-\pi) \beta U\left(\dot{c}^{i}(1), \dot{c}^{i+1}(1)\right)
$$


For each $i \in K$, the extended expected utility function for the initial old agent of type $i$ is a function $\tilde{U}_{0}^{i}: \Re_{+}^{6} \longrightarrow \Re$ defined by,

for all $\left((\dot{\mathbf{c}}(s))_{s \in S}\right) \in \Re_{+}^{6}$,

$$
\tilde{U}_{0}^{i}\left((\dot{\mathbf{c}}(s))_{s \in S}\right) \equiv \pi \beta U\left(\dot{c}^{i}(0), \dot{c}^{i+1}(0)\right)+(1-\pi) \beta U\left(\dot{c}^{i}(1), \dot{c}^{i+1}(1)\right) .
$$

DEFINITION 5.6: An allocation plan $\xi^{*}$ is said to be conditionally Pareto superior to an allocation plan $\xi$ if the following two conditions are satisfied.

(1) For any $t \in \mathcal{N}$, for any $h_{t}=\left(s_{1}, s_{2}, \cdots, s_{t}\right) \in H_{t}$ and for any $i \in K$,

$$
\tilde{U}^{i}\left(\xi_{t}^{*}\left(h_{t}, i\right),\left(\dot{\xi}_{t+1}^{*}\left(\left(h_{t}, s\right), i\right)\right)_{s \in S}\right) \geq \tilde{U}^{i}\left(\xi_{t}\left(h_{t}, i\right),\left(\dot{\xi}_{t+1}\left(\left(h_{t}, s\right), i\right)\right)_{s \in S}\right)
$$

and for any $i \in K$,

$$
\tilde{U}_{0}^{i}\left(\left(\dot{\xi}_{t+1}^{*}(s, i)\right)_{s \in S}\right) \geq \tilde{U}_{0}^{i}\left(\left(\dot{\xi}_{t+1}(s, i)\right)_{s \in S}\right) .
$$

(2) For some $t \in \mathcal{N}$, for some $h_{t}=\left(s_{1}, s_{2}, \cdots, s_{t}\right) \in H_{t}$ or for some $i \in K$,

$$
\tilde{U}^{i}\left(\xi_{t}^{*}\left(h_{t}, i\right),\left(\dot{\xi}_{t+1}^{*}\left(\left(h_{t}, s\right), i\right)\right)_{s \in S}\right)>\tilde{U}^{i}\left(\xi_{t}\left(h_{t}, i\right),\left(\dot{\xi}_{t+1}\left(\left(h_{t}, s\right), i\right)\right)_{s \in S}\right)
$$

or for some $i \in K$,

$$
\tilde{U}_{0}^{i}\left(\left(\dot{\xi}_{t+1}^{*}(s, i)\right)_{s \in S}\right)>\tilde{U}_{0}^{i}\left(\left(\dot{\xi}_{t+1}(s, i)\right)_{s \in S}\right)
$$

DEFINITION 5.7: A feasible allocation plan $\xi$ is said to be conditionally Pareto optimal if there does not exist another feasible allocation plan which is conditionally Pareto superior to $\xi$.

\section{ASSUMPTION 5.1:}

(b1) $U: \Re_{+}^{2} \longrightarrow \Re$ is continuous.

(b2) $U$ is strictly monotone and strictly concave. ${ }^{(2)}$

THEOREM 5.1: A stationary monetary equilibrium is conditionally Pareto optimal under the assumptions (b1) and (b2).

PROOF: In Appendix.

Q.E.D.

(2) If we weaken the strict concavity to the concavity, then one can easily get a counter example which asserts that there is a feasible allocation plan which is conditionally Pareto superior to a stationary monetary equilibrium allocation plan. 


\section{Conclusion}

This paper has studied a stationary, pure exchange overlapping generations model with pairwise trading. This model is to be compared with the usual overlapping generations model - the same model with centralized trading.

In the pairwise version, it has been shown that a standard boundary condition on preferences is sufficient in order for there to be an equilibrium with valued fiat money. In the centralized version, stringent conditions on preferences, endowments, and intertemporal technologies are required in order for there to be such an equilibrium. For example, consider the simple case $w=\dot{w}=1, \beta=1$. It is easy to show that no monetary equilibrium exists in the centralized version. (In this case, the form of the utility function and the endowment are the same when an agent is young and old and the discount factor is one. Therefore, at a zero real interest rate each agent wants to consume the same amount of goods when young and old and to save nothing, i.e., fiat money is not demanded.) Also, it has been shown that some pairwise versions are consistent with some assets bearing a higher rate of return than fiat money. In the centralized version, even if a monetary equilibrium exists, there in no discrepancy between the own rate of return on fiat money and the own rate of return on other assets. Therefore, by introducing pairwise trading, we can get both a robust monetary equilibrium and possible discrepancies between the own rate of return on fiat money and the own rate of return on other assets. 


\section{Appendix}

PROOF OF Theorem 5.1: We obtained the stationary monetary equilibrium as the set of choice variables of agents $\left\{c^{*}, 0, \dot{c}^{*}, 0, \dot{w}, m^{*}, M, b\right\}$, the set of prices $\left\{p^{*}, p^{* *}\right\}$ and the rate of return of the claim $r^{*}$. From this, the stationary monetary equilibrium allocation plan $\xi^{M}$ can be defined as follows. For all $t \in \mathcal{N}$

$$
\left\{\begin{array}{lll}
\xi_{t}^{M}\left(h_{t}, 1\right)=\left(c^{*}, 0,0\right) & \text { for all } h_{t} \in H_{t}, & \\
\xi_{t}^{M}\left(h_{t}, 2\right)=\left(0, c^{*}, 0\right) & \text { for all } h_{t} \in H_{t}, & \\
\xi_{t}^{M}\left(h_{t}, 3\right)=\left(0,0, c^{*}\right) & \text { for all } h_{t} \in H_{t}, & \\
\dot{\xi}_{t}^{M}\left(h_{t}, 1\right)=\left(\dot{c}^{*}, 0,0\right) & \text { for all } h_{t}=\left(s_{1}, s_{2}, \cdots, s_{t}\right) \in H_{t} & \text { with } s_{t}=0 \\
\dot{\xi}_{t}^{M}\left(h_{t}, 2\right)=\left(0, \dot{c}^{*}, 0\right) & \text { for all } h_{t}=\left(s_{1}, s_{2}, \cdots, s_{t}\right) \in H_{t} & \text { with } s_{t}=0, \\
\dot{\xi}_{t}^{M}\left(h_{t}, 3\right)=\left(0,0, \dot{c}^{*}\right) & \text { for all } h_{t}=\left(s_{1}, s_{2}, \cdots, s_{t}\right) \in H_{t} & \text { with } s_{t}=0, \\
\dot{\xi}_{t}^{M}\left(h_{t}, 1\right)=\left(\dot{w}, m^{*}, 0\right) & \text { for all } h_{t}=\left(s_{1}, s_{2}, \cdots, s_{t}\right) \in H_{t} & \text { with } s_{t}=1, \\
\dot{\xi}_{t}^{M}\left(h_{t}, 2\right)=\left(0, \dot{w}, m^{*}\right) & \text { for all } h_{t}=\left(s_{1}, s_{2}, \cdots, s_{t}\right) \in H_{t} & \text { with } s_{t}=1, \\
\dot{\xi}_{t}^{M}\left(h_{t}, 3\right)=\left(m^{*}, 0, \dot{w}\right) & \text { for all } h_{t}=\left(s_{1}, s_{2}, \cdots, s_{t}\right) \in H_{t} & \text { with } s_{t}=1
\end{array}\right\}
$$

Define a function $F:\left[0, c^{*}\right] \times \Re_{+} \longrightarrow \Re_{+}$as

$$
F(\delta, \varepsilon) \equiv U\left(c^{*}-\delta, 0\right)+\pi \beta U\left(\dot{c}^{*}+\varepsilon \delta, 0\right)+(1-\pi) \beta U\left(\dot{w}, m^{*}+\varepsilon \delta\right) .
$$

Then $F$ is continuous on $\left[0, c^{*}\right] \times \Re_{+}$. Let $V \equiv F(0,0) . V$ is the expected value of utility for the agent evaluated at the stationary monetary allocation plan $\xi^{M}$. We will prove the following claim.

Claim $A .1$ : For any $\delta \in\left(0, c^{*}\right], F(\delta, 1)<V$.

Proof of Claim A.1: Pick $\delta \in\left(0, c^{*}\right)$ arbitrarily. Then it is possible to achieve the expected utility level $F(\delta, 1)$ for each agent but it is not chosen. Thus $F(\delta, 1) \leq V$. From the strict concavity of $F(\cdot, 1)$, the optimal choice is unique. Hence $F(\delta, 1)<V$.

End of proof (Claim A.1)

For each $\alpha \in\left(0, c^{*}\right]$, define a set $\Gamma(\alpha) \subset\left[\alpha, c^{*}\right]$ as

$$
\Gamma(\alpha) \equiv\left\{\delta \in\left[\alpha, c^{*}\right] \mid \text { there exists } \varepsilon>1 \text { such that } F(\delta, \varepsilon)=V\right\} .
$$

We will prove the following claim.

Claim A.2: For any $\alpha \in\left(0, c^{*}\right]$, there exists $\bar{\varepsilon}(\alpha)>1$ which satisfies the following. For any $\delta \in \Gamma(\alpha)$ and for any $\varepsilon \in(1, \infty)$,

$$
[F(\varepsilon, \delta)=V] \Longrightarrow[\varepsilon \geq \bar{\varepsilon}(\alpha)]
$$

Proof of Claim A.2: $\quad$ Pick $\alpha \in\left(0, c^{*}\right]$ arbitrarily. Suppose the assertion of the claim is not true. Construct a sequence $\left\{\nu_{k}\right\}_{k=1}^{\infty}$ as $\nu_{k} \equiv 1+\frac{1}{k}$ for all $k=1,2, \ldots$. Then there exist a sequence $\left\{\delta_{k}\right\}_{k=1}^{\infty}$ in $\Gamma(\alpha)$ and a sequence $\left\{\varepsilon_{k}\right\}_{k=1}^{\infty}$ in $(1, \infty)$ which satisfy $F\left(\varepsilon_{k}, \delta_{k}\right)=V$ and $\varepsilon_{k}<\nu_{k}$ for all $k=1,2, \cdots$. We also obtain that $\varepsilon_{k} \rightarrow 1$ as $k \rightarrow \infty$. Since $\Gamma(\alpha)$ is bounded, without loss of generality, we may assume that $\delta_{k} \rightarrow \bar{\delta} \in \mathrm{cl} \Gamma(\alpha)^{(3)}$

(3) $\operatorname{cl} \Gamma(\alpha)$ is the closure of $\Gamma(\alpha)$. 
as $k \rightarrow \infty . F$ is continuous on $\left[0, c^{*}\right] \times \Re_{+}$and $\bar{\delta} \in\left[0, c^{*}\right]$. Therefore $F(\bar{\delta}, 1)=V$. Since $\bar{\delta} \in\left[\alpha, c^{*}\right]$ with $\alpha>0, F(\bar{\delta}, 1)<V$ by Claim $A .1$. This is a contradiction.

End of proof (Claim A.2)

Now we are ready to start to prove the assertion of the theorem. By way of contradiction, suppose there exists a feasible allocation plan $\xi^{\dagger}$ which is conditionally Pareto superior to $\xi^{M}$. For $t \in \mathcal{N}$, define a function $\delta_{t}: H_{t} \times K \longrightarrow \Re_{+}^{3}$ as

$$
\delta_{t} \equiv \xi_{t}^{M}-\xi_{t}^{\dagger}
$$

and define a function $\dot{\delta}_{t}: H_{t} \times K \longrightarrow \Re_{+}^{3}$ as

$$
\dot{\delta}_{t} \equiv \dot{\xi}_{t}^{M}-\dot{\xi}_{t}^{\dagger}
$$

Without loss of generality, we may assume that the allocation plan $\xi^{\dagger}$ satisfies feasibility condition with equality. Therefore, for any $t \in \mathcal{N}, \delta_{t}=-\dot{\delta}_{t}$. First, we will prove the following claim.

Claim A.3: For any $i \in K$, for any $t \in \mathcal{N}$ and for any $h_{t} \in H_{t}, \delta_{t, i+1}\left(h_{t}, i\right)^{(4)}=\delta_{t, i+2}\left(h_{t}, i\right)=0$

Proof of Claim A.3: Pick any $i \in K$, any $t \in \mathcal{N}$ and any $h_{t} \in H_{t}$ arbitrarily. Consider the situation that the young agent of type $i$ meets the old agent of type $i$ or type $i+2$. Since neither the agent of type $i$ nor the agent of type $i+2$ is endowed with good $i+1, \delta_{t, i+1}\left(h_{t}, i\right)=0$. Since agents of type $i$ cannot derive utility from good $i+2$, without loss of generality we may assume $\xi_{i, i+2}^{\dagger}\left(h_{t}, i\right)=0$. Therefore, from $\xi_{t, i+2}^{M}\left(h_{t}, i\right)=0$, we obtain $\delta_{t, i+2}\left(h_{t}, i\right)=0$.

End of proof (Claim A.3)

Secondly, we will prove the following claim.

Claim A.4: For any $i \in K$, for any $t \in \mathcal{N}$ and for any $h_{t} \in H_{t}$,

$$
\left[\delta_{t, i}\left(h_{t}, i\right)>0\right] \Longrightarrow\left[\delta_{t, i}\left(h_{t}, i\right) \in \Gamma\left(\delta_{t, i}\left(h_{t}, i\right)\right)\right]
$$

Moreover, for any $t^{\prime} \in \mathcal{N}$ and for any $h_{t^{\prime}}^{\prime} \in H_{t^{\prime}}$,

$$
\left[\delta_{t^{\prime}, i}\left(h_{t^{\prime}}^{\prime}, i\right) \geq \delta_{t, i}\left(h_{t}, i\right)\right] \Longrightarrow\left[\delta_{t^{\prime}, i}\left(h_{t^{\prime}}^{\prime}, i\right) \in \Gamma\left(\delta_{t, i}\left(h_{t}, i\right)\right)\right]
$$

Proof of Claim A.4: Pick any $i \in K$, any $t \in \mathcal{N}$ and any $h_{t} \in H_{t}$ arbitrarily. Assume $\delta_{t, i}\left(h_{t}, i\right)>0$. Let

$$
V^{\dagger} \equiv \tilde{U}^{i}\left(\xi_{t}^{\dagger}\left(h_{t}, i\right), \dot{\xi}_{t+1}^{\dagger}\left(\left(h_{t}, 0\right), i\right), \dot{\xi}_{t+1}^{\dagger}\left(\left(h_{t}, 1\right), i\right)\right)
$$

$V^{\dagger}$ is the expected value of utility evaluated at the allocation plan $\xi^{\dagger}$ for the young agent of type $i$ in period $t$ when a history $h_{t}$ is realized. Then, by Claim $A .3$ and the definition of $\delta_{t}$, we obtain

$V^{\dagger}=U\left(c^{*}-\delta_{t, i}\left(h_{t}, i\right), 0\right)+\pi \beta U\left(\dot{c}^{*}+\delta_{t+1, i}\left(\left(h_{t}, 0\right), i\right), 0\right)$

(4) $\delta_{t, i}(\cdot)$ is a projection of $\delta_{t}(\cdot)$ with respect to the $i$-th argument. This principle is applied to other functions such as $\dot{\delta}_{t}(\cdot), \xi_{t}(\cdot)$ and $\dot{\xi}_{t}(\cdot)$. 


$$
+(1-\pi) \beta U\left(\dot{w}, m^{*}+\delta_{t+1, i+1}\left(\left(h_{t}, 1\right), i\right)\right)
$$

If $\delta_{t, i}\left(h_{t}, i\right)>c^{*}$, then $\xi_{t, i}^{\dagger}\left(h_{t}, i\right)<0$. Since consumption is non-negative, this is a contradiction. Therefore we obtain $0<\delta_{t, i}\left(h_{t}, i\right) \leq c^{*}$. By way of contradiction, suppose $\delta_{t, i}\left(h_{t}, i\right) \notin \Gamma\left(\delta_{t, i}\left(h_{t}, i\right)\right)$. This means that $F\left(\delta_{t, i}\left(h_{t}, i\right), \varepsilon\right) \neq V$ for any $\varepsilon>1$. Suppose $F\left(\delta_{t, i}\left(h_{t}, i\right), \varepsilon^{\prime}\right)>V$ for some $\varepsilon^{\prime}>1$. By Claim A.1, $F\left(\delta_{t, i}\left(h_{t}, i\right), 1\right)<V$. Since $F$ is continuous, there exists some $\varepsilon^{*}>1$ such that $F\left(\delta_{t, i}\left(h_{t}, i\right), \varepsilon^{*}\right)=V$. This is a contradiction. Therefore we obtain,for any $\varepsilon>1, F\left(\delta_{t, i}\left(h_{t}, i\right), \varepsilon\right)<V$, i.e.,

$$
U\left(c^{*}-\delta_{t, i}\left(h_{t}, i\right), 0\right)+\pi \beta U\left(\dot{c}^{*}+\varepsilon \delta_{t, i}\left(h_{t}, i\right)\right)+(1-\pi) \beta U\left(\dot{w}, m^{*}+\varepsilon \delta_{t, i}\left(h_{t}, i\right)\right)<V
$$

Since $\xi^{\dagger}$ is conditionally Pareto superior to $\xi^{M}$, we obtain $V^{\dagger} \geq V$. Therefore, from $(A 1)$ and (A2), for any $\varepsilon>1$,

$$
\begin{gathered}
U\left(c^{*}-\delta_{t, i}\left(h_{t}, i\right), 0\right)+\pi \beta U\left(\dot{c}^{*}+\delta_{t+1, i}\left(\left(h_{t}, 0\right), i\right), 0\right)+(1-\pi) \beta U\left(\dot{w}, m^{*}+\delta_{t+1, i+1}\left(\left(h_{t}, 1\right), i\right)\right)> \\
U\left(c^{*}-\delta_{t, i}\left(h_{t}, i\right), 0\right)+\pi \beta U\left(\dot{c}^{*}+\varepsilon \delta_{t, i}\left(h_{t}, i\right), 0\right)+(1-\pi) \beta U\left(\dot{w}, m^{*}+\varepsilon \delta_{t, i}\left(h_{t}, i\right)\right) .
\end{gathered}
$$

Hence, from the strict monotonicity of the utility function, we obtain, for any $\varepsilon>1$,

$$
\delta_{t+1, i}\left(\left(h_{t}, 0\right), i\right)>\varepsilon \delta_{t, i}\left(h_{t}, i\right) \quad \text { or } \quad \delta_{t+1, i+1}\left(\left(h_{t}, 1\right), i\right)>\varepsilon \delta_{t, i}\left(h_{t}, i\right)
$$

This is a contradiction. This completes the proof of the first half of the claim. Pick any $t^{\prime} \in \mathcal{N}$ and any $h_{t^{\prime}}^{\prime} \in H_{t^{\prime}}$ arbitrarily. Then, by the same way, we can show that $\delta_{i, t^{\prime}}\left(h_{t^{\prime}}^{\prime}, i\right) \leq c^{*}$. Thus $\delta_{t^{\prime}, i}\left(h_{t^{\prime}}^{\prime}, i\right) \geq \delta_{t, i}\left(h_{t}, i\right)$ implies $\delta_{t, i}\left(h_{t}, i\right) \leq \delta_{t^{\prime}, i}\left(h_{t^{\prime}}^{\prime}, i\right) \leq c^{*}$. Then, by the same way, we can prove the second half of the claim.

End of proof (Claim A.4)

Thirdly, we will prove the following claim.

Claim A.5: For any $\alpha \in\left(0, c^{*}\right]$, there exists $\bar{\varepsilon}(\alpha)>1$ which satisfies the following. For any $i \in K$, for any $t \in \mathcal{N}$ and for any $h_{t} \in H_{t}$,

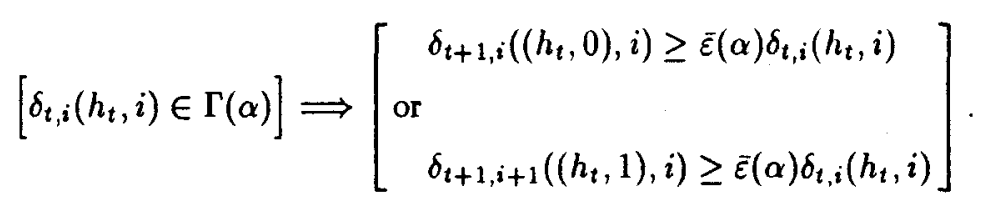

Proof of Claim A.5: Pick any $\alpha \in\left(0, c^{*}\right]$ arbitrarily. Then, by Claim $A .2$, there exists $\bar{\varepsilon}(\alpha)>1$ which satisfies the following. For any $\delta \in \Gamma(\alpha)$ and for any $\varepsilon \in(1, \infty)$,

$$
[F(\varepsilon, \delta)=V] \Longrightarrow[\varepsilon \geq \bar{\varepsilon}(\alpha)]
$$

Pick any $i \in K$, any $t \in \mathcal{N}$ and any $h_{t} \in H_{t}$ arbitrarily. Assume $\delta_{t, i}\left(h_{t}, i\right) \in \Gamma(\alpha)$. Then, by the definition of $\Gamma(\alpha)$, there exists some $\varepsilon>1$ which satisfies that $F\left(\delta_{t, i}\left(h_{t}, i\right), \varepsilon\right)=V$. Then, by the above argument, $\varepsilon \geq \bar{\varepsilon}(\alpha)$. Thus we obtain

$$
\begin{gathered}
U\left(c^{*}-\delta_{t, i}\left(h_{t}, i\right), 0\right)+\pi \beta U\left(\dot{c}^{*}+\delta_{t+1, i}\left(\left(h_{t}, 0\right), i\right), 0\right)+(1-\pi) \beta U\left(\dot{w}, m^{*}+\delta_{t+1, i+1}\left(\left(h_{t}, 1\right), i\right), 0\right)= \\
\left.\left.U\left(c^{*}-\delta_{t, i}\left(h_{t}, i\right), 0\right)+\pi \beta U\left(\dot{c}^{*}+\varepsilon \delta_{t, i}\left(h_{t}, i\right), 0\right), 0\right)+(1-\pi) \beta U\left(\dot{w}, m^{*}+\varepsilon \delta_{t, i}\left(h_{t}, i\right), 0\right), 0\right) \geq
\end{gathered}
$$




$$
\left.\left.U\left(c^{*}-\delta_{t, i}\left(h_{t}, i\right), 0\right)+\pi \beta U\left(\dot{c}^{*}+\bar{\varepsilon}(\alpha) \delta_{t, i}\left(h_{t}, i\right), 0\right), 0\right)+(1-\pi) \beta U\left(\dot{w}, m^{*}+\bar{\varepsilon}(\alpha) \delta_{t, i}\left(h_{t}, i\right), 0\right), 0\right) .
$$

Hence, from the strict monotonicity of the utility function, we obtain

$$
\delta_{t+1, i}\left(\left(h_{t}, 0\right), i\right) \geq \bar{\varepsilon}(\alpha) \delta_{t, i}\left(h_{t}, i\right) \quad \text { or } \quad \delta_{t+1, i+1}\left(\left(h_{t}, 1\right), i\right) \geq \bar{\varepsilon}(\alpha) \delta_{t, i}\left(h_{t}, i\right)
$$

End of proof (Claim A.5)

Now we are finally on the stage to prove the assertion of the theorem. First, if for all $i \in K$, for all $t \in \mathcal{N}$ and for all $h_{t} \in H_{t}, \delta_{t}\left(h_{t}, i\right)=0$, then this means for all $i \in K$, for all $t \in \mathcal{N}$ and for all $h_{t} \in H_{t}$, $\dot{\delta}_{t}\left(h_{t}, i\right)=0$. Therefore we obtain $\xi^{M}=\xi^{\dagger}$. This contradicts that $\xi^{\dagger}$ conditionally Pareto dominates $\xi^{M}$. Secondly, if for all $i \in K$, for all $t \in \mathcal{N}$ and for all $h_{t} \in H_{t}, \delta_{t}\left(h_{t}, i\right) \leq 0,{ }^{(5)}$ and for some $i \in K$, for some $t \in \mathcal{N}$ and for some $h_{t} \in H_{t}, \delta_{t}\left(h_{t}, i\right)<0$, then some initial old agent is worse off. This is also a contradiction. Therefore, there exists some $\bar{\imath} \in K$ and $\bar{h}_{\bar{t}} \in H_{\bar{i}}$ for some $\bar{t} \in \mathcal{N}$ such that $\delta_{\bar{t}}\left(\bar{h}_{\bar{t}}, \bar{i}\right)>0$. Then, by Claim $A .3$, we obtain

$$
\delta_{\bar{t}, \bar{i}}\left(\bar{h}_{\bar{t}}, \bar{\imath}\right)>0 \text {. }
$$

Then, by Claim $A .4, \delta_{\bar{t}_{\bar{t}}}\left(\bar{h}_{\bar{t}}, \bar{\imath}\right) \in \Gamma\left(\delta_{\bar{t}_{,},}\left(\bar{h}_{\bar{t}}, \bar{\imath}\right)\right)$. Then, by Claim A.5, there exists $\bar{\varepsilon}>1$ which satisfies

$$
\delta_{\bar{t}+1, \bar{z}}\left(\left(\bar{h}_{\bar{t}}, 0\right), \bar{\imath}\right) \geq \bar{\varepsilon} \delta_{\bar{t}, \bar{i}}\left(\bar{h}_{\bar{t}}, \bar{\imath}\right)
$$

or

$$
\delta_{\bar{t}+1, \bar{i}+1}\left(\left(\bar{h}_{\bar{t}}, 1\right), \bar{\imath}\right) \geq \bar{\varepsilon} \delta_{\bar{t}, \bar{i}}\left(\bar{h}_{\bar{t}}, \bar{\imath}\right)
$$

In the case that $(A 4)$ holds, we obtain $\delta_{\bar{t}+1, \bar{i}}\left(\left(\bar{h}_{\bar{t}}, 0\right), \bar{i}\right)>\delta_{\bar{i}, \bar{i}}\left(\bar{h}_{\bar{i}}, \bar{\imath}\right)$. Then, by Claim $A .4$, we obtain $\delta_{\bar{t}+1, \bar{i}}\left(\left(\bar{h}_{\bar{t}}, 0\right), \bar{\imath}\right) \in \Gamma\left(\delta_{\bar{t}, \bar{i}}\left(\bar{h}_{\bar{t}}, \bar{\imath}\right)\right)$. Therefore, by Claim $A .5$, we obtain

$$
\delta_{\bar{t}+2, \bar{i}}\left(\left(\bar{h}_{\bar{t}}, 0,0\right), \bar{\imath}\right) \geq \bar{\varepsilon} \delta_{\bar{t}+1, \bar{i}}\left(\left(\bar{h}_{\bar{t}}, 0\right), \bar{\imath}\right)
$$

or

$$
\delta_{\bar{i}+2, \bar{i}+1}\left(\left(\bar{h}_{\bar{i}}, 0,1\right), \bar{z}\right) \geq \bar{\varepsilon} \delta_{\bar{i}+1, \mathbf{r}}\left(\left(\bar{h}_{\bar{i}}, 0\right), \bar{i}\right) .
$$

Note that, in the above, we used $\bar{\varepsilon}$ which is the same $\bar{\varepsilon}$ in $(A 4)$ and $(A 5)$.

In the case that $(A 5)$ holds, we obtain $\delta_{\bar{t}+1, \bar{i}+1}\left(\left(\bar{h}_{\bar{t}}, 1\right), \bar{\imath}\right)>\delta_{\bar{t}, \bar{i}}\left(\bar{h}_{\bar{i}}, \bar{\imath}\right)$. Then, by the same way, we obtain

$$
\delta_{\bar{t}+2, \bar{i}+1}\left(\left(\bar{h}_{\bar{t}}, 1,0\right), \bar{\imath}+1\right) \geq \bar{\varepsilon} \delta_{\bar{t}+1, \bar{i}+1}\left(\left(\bar{h}_{\bar{i}}, 1\right), \bar{\imath}\right)
$$

or

$$
\delta_{\bar{t}+2, \bar{\imath}+2}\left(\left(\bar{h}_{\bar{t}}, 1,1\right), \bar{\imath}+1\right) \geq \bar{\varepsilon} \delta_{\bar{t}+1, \bar{z}+1}\left(\left(\bar{h}_{\bar{t}}, 1\right), \bar{i}\right) .
$$

Since $\bar{\varepsilon}>1$ it is impossible to continue the above process. This concludes the proof of the theorem.

Q.E.D.

(5) In this paper, we use inequalities for vectors in the following sense. Let $v=\left(v_{1}, v_{2}, \cdots, v_{n}\right) \in \Re^{n}$. $v \geq 0$ means for all $i \in\{1,2, \cdots, n\}, v_{i} \geq 0 . v>0$ means $x \geq 0$ and for some $i \in\{1,2, \cdots, n\}, v_{i}>0$. 


\section{References}

1. A. M. Feldman, Bilateral Trading Processes, Pairwise Optimality, and Pareto Optimality, Rev. Econ. Studies 40 (1973), 463-474.

2. M. Harris, Expectations and Money in a Dynamic Exchange Model, Econometrica 47 (1979), 1403-1419.

3. W. S. Jevons, "Money and the Mechanism of Exchange" Appleton, London, 1875.

4. N. Kiyotaki and R. Wright, On Money as a Medium of Exchange, J. Polit. Econ 97 (1989), 927-954.

5. J. M. Ostroy, The informational efficiency of monetary exchange, Amer. Econ. Rev. 63 (1973), 597-610.

6. J. M. Ostroy and R. M. Starr, Money and the Decentralization of Exchange, Econometrica 42 (1974), $1093-1113$.

7. D. Peled, "Government Issued Indexed Bonds - Do They Improve Matters?" Ph.D. Thesis, University of Minnesota, 1980.

8. D. Peled, Informational Diversity over Time and the Optimality of Monetary Equilibria, J. Econ Theory 28 (1982), 255-274.

9. Y. Sakai, Conditional Pareto Optimality of Stationary Equilibrium in a Stochastic Overlapping Generations Model, J. Econ Theory 44 (1988), 209-213.

10. R. M. Townsend, Asset-Return Anomalies in a Monetary Economy, J. Econ Theory 41 (1987), 219-247. 